\title{
Generation of large-bandwidth $x$-ray free electron laser with evolutionary many-objective optimization algorithm
}

\author{
Jiawei Yan $^{1,2}$ and Haixiao Deng ${ }^{1,3, *}$ \\ ${ }^{1}$ Shanghai Institute of Applied Physics, Chinese Academy of Sciences, Shanghai 201800, China \\ ${ }^{2}$ University of Chinese Academy of Sciences, Beijing 100049, China \\ ${ }^{3}$ Shanghai Advanced Research Institute, Chinese Academy of Sciences, Shanghai 201210, China
}

(Received 14 June 2018; published 21 February 2019)

\begin{abstract}
X-ray free-electron lasers (XFELs) are cutting-edge scientific instruments for a wide range of disciplines. Conventionally, the narrow bandwidth is pursued in an XFEL. However, in recent years, the large-bandwidth XFEL operation schemes are proposed for x-ray spectroscopy and x-ray crystallography, in which overcompression is a promising scheme to produce broad-bandwidth XFEL pulses through increasing the electron beam energy chirp. In this paper, combining with the beam yaw correction to overcome the transverse slice misalignment caused by the coherent synchrotron radiation, finding out the overcompression working point of the linac is treated as a many-objective (having four or more objectives) optimization problem, thus the nondominated sorting genetic algorithm III (NSGA-III) is applied to the beam dynamic optimization for the first time. Start-to-end simulations demonstrate a full bandwidth of $4.79 \%$ for Shanghai soft x-ray free-electron laser user facility. Furthermore, the NSGA-III is found to be very efficient by providing a set of reference points with preferences. This paves the way for applying this algorithm to online optimization of a real machine.
\end{abstract}

DOI: $10.1103 /$ PhysRevAccelBeams.22.020703

\section{INTRODUCTION}

X-ray free electron lasers (XFELs) are leading-edge instruments in a wide range of research fields that can provide short wavelength radiation with high brightness and ultra-fast time structures [1]. Most worldwide XFEL facilities [2-6] are based on self-amplified spontaneous emission (SASE) [7]. The relative bandwidth of the SASEXFEL pulses at saturation is of the order of the Pierce parameter [8], with values between $10^{-3}$ and $10^{-4}$. To generate fully coherent XFEL pulses, several schemes [9] have been proposed to further decrease the XFEL bandwidth. In addition to narrow bandwidth XFEL pulses, the large-bandwidth XFEL operation has attracted increasing attention. Broad-bandwidth XFEL pulses are very useful in many spectroscopy experiments [10,11], multi-wavelength anomalous diffraction [12,13], and x-ray crystallography $[14,15]$. Furthermore, in the large-bandwidth mode, the XFEL wavelength can be adjusted by simply applying a monochromator without changing any parameters on the accelerator side.

\footnotetext{
*denghaixiao@sinap.ac.cn
}

Published by the American Physical Society under the terms of the Creative Commons Attribution 4.0 International license. Further distribution of this work must maintain attribution to the author(s) and the published article's title, journal citation, and DOI.
According to the FEL resonance condition [8]:

$$
\lambda=\frac{\lambda_{u}}{2 \gamma^{2}}\left(1+\frac{K^{2}}{2}\right)
$$

where $\lambda$ is the FEL wavelength, $\lambda_{u}$ is the undulator period length, $\gamma$ is the mean Lorentz factor of the electrons, and $K$ is the undulator field parameter, the XFEL wavelength is determined by the electron beam energy and the undulator field parameters. In principle, properly sending the headtail transversely tilted electron bunches into a transverse gradient undulator [16] or into a planar undulator with its natural gradient [17] can make different parts of the bunch experience different magnetic field, thus generating broadband FEL pulses. In addition, using electron beams with time-energy correlation is a more natural way to obtain broad-bandwidth FEL, which may be achieved without additional hardware elements in currently existing facilities. The simplest way to obtain the energy chirp is by offcrest acceleration, while it is inefficient and at the cost of reducing beam energy. Utilizing the longitudinal space charge of a strongly compressed electron beam to increase the correlated energy spread was proposed for the European XFEL [18]. There is another special compression mode named overcompression [19-22] that can be used to generate a large energy chirp. In this scheme electron beams are overcompressed in the bunch compressor, which means the head and tail of the bunch will interchange their 
positions. The sign of the energy chirp will also be changed. Thus, the beam energy chirp will be increased by the wakefields of subsequent rf structures. In addition, the corrugated structure insertion can be used to further enhance the energy chirp [23]. The crucial issue of the overcompression mode is to find an appropriate working point of the linac where the electron beams have large energy chirp while other beam qualities can be maintained. The above process can be treated as a many-objective (having four or more objectives) optimization problem in which the energy chirp, peak current, energy spread, current profile, and projected emittance of the electron bunch are objectives.

It is a common strategy to transform multiple objectives into one or two objectives by using the weighted method. Nevertheless, not only it is difficult to determine weights in this scenario, but it also loses the opportunity to analyze the relationships between each objective. Multi-objective evolutionary algorithms (MOEAs) [24], such as nondominated sorting genetic algorithm II (NSGA-II) [25], have been widely and successfully used in the accelerator community [26-30] for optimization problems with two or three objectives. However, in recent years, it has been pointed out that Pareto-dominance based MOEAs will encounter some difficulties in many-objective optimization problems (MaOPs) $[31,32]$. With the number of objectives increases, the proportion of nondominated elements in the population is close to one, which makes it very difficult for the Pareto-dominance based MOEAs to discriminate among solutions utilizing only the dominance relation. Moreover, to approximate the entire Pareto-optimal front properly, the number of solutions needs to be increased exponentially with the number of objective functions, which leads to longer execution time. Recently, an improved NSGA-II procedure, which was termed NSGA-III $[33,34]$, has been proposed as an evolutionary many-objective algorithm. NSGA-III maintains the diversity among population members by supplying and adaptively updating a set of well-spread reference points. It has been demonstrated that NSGA-III is efficient in optimizing 2 to 15 objectives problems [35-37]. In this paper, using the Shanghai soft $\mathrm{x}$-ray free-electron laser (SXFEL) user facility parameters, the overcompression process is optimized by adjusting accelerator operation parameters with NSGA-III to explore the maximum FEL bandwidth.

Improving efficiency is a common challenge of applying evolutionary many-objective or multi-objective optimization algorithms to online experiments. The main reason is that online experiments cannot be run in parallel like simulation, which leads to a lot of time to find the Paretooptimal front. One of the advantages of the NSGA-III is that it can find part of the Pareto-optimal front rather than the entire Pareto-optimal front by supplying reference points with preferences, which makes this algorithm very efficient. Therefore, the preference-based NSGA-III is also used to optimize the large-bandwidth mode and its performance is compared with the widely used NSGA-II.

The paper is structured as follows. In Sec. II, the optimization methods of the overcompression mode for SXFEL user facility are described, including the optimization strategy and the algorithm used. The optimization results of NSGA-III and three typical cases are shown in Sec. III. Results of the NSGA-III with preferred reference points are presented in Sec. IV. The conclusions and outlook are summarized in Sec. V.

\section{METHODS}

\section{A. Optimization strategy}

To obtain high quality electron beams in the over compression mode, electron bunch parameters that are important for XFEL lasing including the peak current, slice energy spread, energy chirp, current profile, and projected emittance are selected as optimization objectives. In this optimization, the start-to-end simulations are performed to compute these optimization objectives and validate the largebandwidth XFEL generation in the undulator. ASTRA [38] is used to track electron beams in the injector where the transverse space charge forces are strong. Tracking simulation in the main linac is performed by the ELEGANT [39] and its parallel version PELEGANT [40] in which collective effects like the coherent synchrotron radiation (CSR), longitudinal space charge, and wakefields are considered. The calculation of objectives is based on the PELEGANT simulation results. Thus, to balance the calculation accuracy and time spent, the PELEGANT simulation is performed with one hundred thousand macroparticles during the optimization. GENESIS [41] is used to verify the XFEL generation.

An electron bunch from the PELEGANT simulation result is divided into 100 slices to calculate objective values. The peak current $I_{\max }$ is defined as the maximum current value of these slices. $2 \%$ of the $I_{\max }$ is chosen as a cutoff value. The cutoff slices on two sides of $a$ bunch are $a$ and $b$ respectively. The objective value of the energy spread, $\delta_{\text {mean }}$, is defined as average slice energy spread of slices between the two cutoff slices:

$$
\delta_{\text {mean }}=\frac{\sum_{a}^{b} \delta_{i}}{b-a+1},
$$

where $\delta_{i}$ represents the energy spread of the ith slice. The energy chirp is defined as the relative energy difference between the two cutoff slices:

$$
\sigma_{d}=\frac{\left|\gamma_{b}-\gamma_{a}\right|}{\frac{1}{b-a+1} \sum_{a}^{\mathrm{b}} \gamma_{i}}
$$

where $\gamma_{i}$ is the energy of the ith slice. To describe the current profile of an electron beam, a profile factor, $C$, is defined as the ratio of the sum of central 50 slice current to the sum of total slice current: 


$$
C=\frac{\sum_{26}^{75} I_{i}}{\sum_{1}^{100} I_{i}}
$$

It should be pointed out that the profile factor does not describe a specific shape. This is to analyze the relationship between the current profile and energy chirp during the optimization. In order to consider the effect of CSR on the bending plane, the projected emittance $\varepsilon_{x}$ is defined as the normalized horizontal emittance at the exit of the linac.

These optimization goals are not independent of each other. For example, peak current and slice energy spread are two inherently conflicting objectives. Besides that, the current profile will influence the energy chirp brought by longitudinal wakefields. Therefore, it is important for the overcompression mode to optimize all the objectives at the same time. As mentioned before, those widely used MOEAs are originally proposed for problems with two or three objectives. In recent years, numerous evolutionary manyobjective optimization algorithms have been put forward to handle those problems with more than three objectives. The NSGA-III, one of the most frequently-used many-objective optimization algorithm, is applied to optimize the overcompression mode in SXFEL user facility. It is more convenient for the algorithm when all the objectives have the same optimization direction and sign. Thus, in the actual optimization, $-\sigma_{d},-C,-\delta_{\operatorname{mean}}^{-1},-I_{\max }$, and $-\epsilon_{x}^{-1}$ are treated as the objective functions and simultaneously minimized.

\section{B. Algorithm}

In general, Pareto-dominance based MOEAs manipulate a set of solutions (a population) toward the Pareto-optimal front through a set of typical operations including selection, crossover, and mutation. The selection operation applies the evolution pressure by selecting the best solutions in the iteration. The crossover operation combines existing solutions to generate new solutions. The mutation operation produces new solutions by altering the existing solutions. For each iteration (called generation), the crossover or mutation operation creates a new population of equal size (called offspring population) from the existing population with a certain probability. The NSGA-III that used in this study is an improved version of the NSGA-II for MaOPs. The basic framework of NSGA-III is similar to the NSGA-II [25] but the selection operation is quite different. The main procedure of NSGA-III is briefly described as follows. In the beginning, $N$ solutions called the initial population are randomly generated, where $N$ is the population size. The following steps are continuously iterated until the stop criterion is reached. Take the t-th generation as an example. Assume the population is $Q_{t}$. After the tournament selection, polynomial mutation [42], and simulated binary crossover [43], an offspring $P_{t}$ is generated from $Q_{t}$ with the same size. Thereafter, the $Q_{t}$ and $P_{t}$ are merged to $R_{t}$, and the best $N$ members need to be selected from the $R_{t}$ for the next generation. Before the selection, the population $R_{t}$ is sorted into multiple nondominated fronts based on the Pareto dominance [25]. Then, each nondominated fronts is selected one at a time to construct a new population $S_{t}$, starting from the first nondominated fronts, until the size of $S_{t}$ is larger than or equal to the population size. The last front included is defined as the $M$-th Pareto-optimal front. Solutions from one to $(M-1)$ fronts will be selected for the next generation and the rest individuals are chosen from the $M$ th Pareto-optimal front one by one, until the size of next generation population is equal to $N$. In NSGA-II, solutions with large crowding distance will be chosen. The crowding distance of a solution is defined as the average distance of its surrounding solutions, which is not working well for the MaOPs [44]. In NSGA-III, a new selection mechanism based on a set of supplied reference points is proposed to analyze solutions in the $M$ th Pareto-optimal front more systematically.

The reference points used in the NSGA-III are predefined to ensure the diversity of the population. These reference points can either be generated in a structured manner or chosen based on any preference information. In the selection, objectives and the reference points are normalized to be in the same range. After the normalization, the zero vector is treated as the ideal point of the $S_{t}$ and the reference lines are defined by joining the ideal point with reference points. Thereafter, the perpendicular distances between a solution and each reference line are calculated. Every population member in the $S_{t}$ is associated with a reference point corresponding to the minimum perpendicular distance. Then, the number of solutions associated with each reference point is treated as the niche count. Some solutions from the $M$ th Pareto-optimal front of the $S_{t}$ are included into the next population based on the reference point niche count. More details about the NSGA-III can be found in [33].

The Das and Dennis's [45] systematic approach used in the original NSGA-III paper [33] is also used to generate reference points in this optimization. The reference points generated by this method incline to all objectives equally.

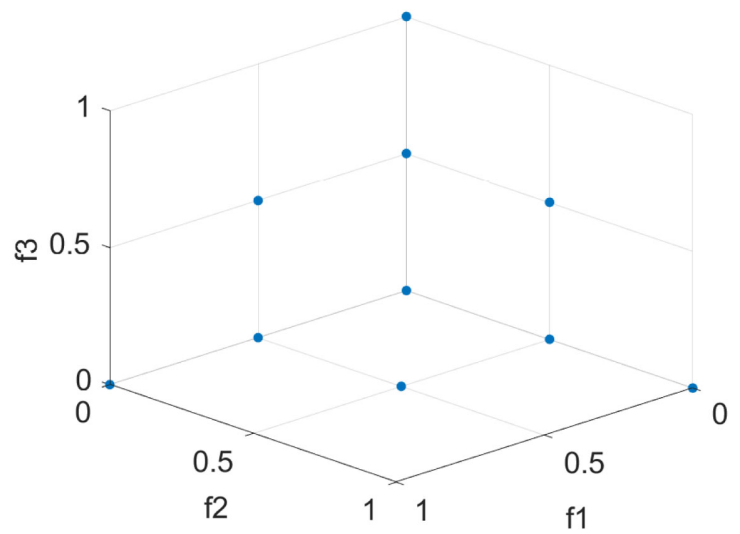

FIG. 1. The distribution of the reference points of a fiveobjective problem with $p=2$ in the space constituted by any three of the five objective functions. 
If $p$ divisions are considered for each objective in an $K$ objective problem, the total number of reference points $H$ is given by: $H=C_{p+K-1}^{K-1}$. For example, in a five-objective problem, two divisions $(p=2)$ are chosen for each objective axis, 15 reference points will be created. Figure 1 shows the distribution of these reference points in the space constituted by any three of the five objective functions. In this optimization, the $p$ is chosen to be 7 to handle the 5 objectives so the total number of reference points is 330 .

\section{RESULTS OF SXFEL USER FACILITY}

\section{A. The SXFEL user facility}

As the first X-ray FEL in China, the SXFEL user facility is under construction at Shanghai [46]. The SXFEL user facility will be equipped with two undulator lines. One is the two-stage seeded FEL line to generate $3 \mathrm{~nm}$ fully coherent FEL pulses, and the other is the SASE line aimed at $2 \mathrm{~nm}$ using an in-vacuum undulator. The scope of the optimization in this study is to design the large-bandwidth operation mode for the SASE line.

In the baseline design of the SXFEL user facility, $0.5 \mathrm{nC}$ electron bunches with $130 \mathrm{MeV}$ are generated in the injector section which includes two S-band accelerating structures and a laser heater. Downstream of the injector is the main linac with three accelerating sections and two bunch compressors. Electron beams are accelerated at the off-crest phase of a S-band accelerating section to create an energy chirp and an X-band rf cavity is used to linearize the chirp. Following this, electron beams with $256 \mathrm{MeV}$ are compressed in the first magnetic chicane. There are two C-band linac accelerators are used to further increase the beam energy to $1.5 \mathrm{GeV}$. The second bunch compressor is between the two C-band accelerating sections to further compress electron beams and obtain peak currents larger than $700 \mathrm{~A}$. Finally, electron bunches are sent to the two FEL lines. In the overcompression mode, energy chirp is increased by the longitudinal wakefields after the overcompression. Therefore, it is more appropriate to make the electron beam be overcompressed in the first bunch compressor and turn off the second bunch compressor to utilize more longitudinal wakefields. Layout of the main linac with a single-stage bunch compressor and the SASE line are shown in Fig. 2.

The angle of the first bunch compressor $\left(\theta_{B C 1}\right)$, voltages and phases of the $\mathrm{S}$-band accelerating section $\left(V_{1}, \varphi_{1}\right)$ and the X-band linearizer $\left(V_{x}, \varphi_{x}\right)$ are selected as optimization variables. The ranges of these variables are decided by the limits of related hardware. In addition, overcompressed electron bunches have gone through full compression status in chicane where the CSR becomes quite strong [47]. The strong CSR brings electron bunches with a longitudinally dependent energy loss which will be turned into slice misalignment called beam yaw and increase the projected emittance of the bending plane. When there is no additional correction, the beam yaw will be further increased in the subsequent accelerating sections due to the transverse wakefields. Recently, the dispersion section based beam yaw correction has been proposed and experimentally verified $[48,49]$. This scheme removes the beam yaw by sending energy chirped electron beams into well-controlled dispersion sections. In the SXFEL user facility, there are two quadrupoles in the first magnetic chicane that can be used to correct the first order beam yaw. To maximize the energy chirp and eliminate the beam yaw caused by the CSR simultaneously, the strength of the two quadrupoles in the first bunch compressors is added to optimization variables. The second order beam yaw correction needs additional sextupole magnets, which is not considered in this optimization.

As mentioned earlier, in the many-objective optimization problem, a large number of solutions are needed to describe the entire Pareto-optimal front. Therefore it is necessary to concentrate the entire population in the region of interest. Rejecting infeasible solutions during the optimization, such as artificially altering their objective values, is the most effective way to handle constraints and has been widely used in beam dynamic optimization. However, it should be noted that rejecting a lot of solutions may lead to the loss of population diversity and make the algorithm prone to be trapped in local optima [50]. Hence, setting objective constraints usually requires some trials in advance to avoid rejecting too many solutions. In this optimization, considering the single-stage bunch compression, those solutions with a peak current more than $2000 \mathrm{~A}$ or less than $700 \mathrm{~A}$ are artificially given the worst objective values. In addition, in order to focus the optimization on finding the optimal solutions in the overcompression region, those electron bunches which are not overcompressed, i.e., the tail of the electron bunch with larger energy than the head, will also be given the worst objective values.

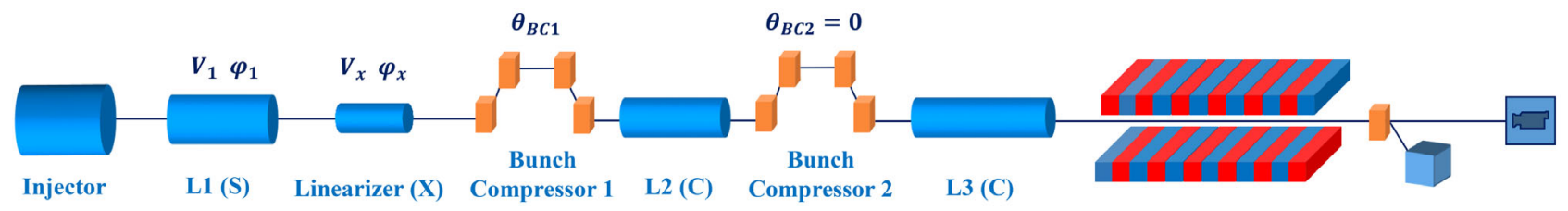

FIG. 2. Layout of the SXFEL user facility linac, with the injector section, S-band sections (L1), one X-band linearizer (LX), C-band sections (L2, L3), and two bunch compressor chicanes (BC1, BC2). Angle of the BC1, voltages, and phases of L1 and LX are optimized for the large-bandwidth operation mode. The second bunch compressor is turned off to utilize more longitudinal wakefields. 
Since only the parameters in the main accelerator are optimized, the time required to run an optimization is mainly determined by the runtime of the PELEGANT. Suppose the PELEGANT runs once for $t$, the population size i.e., the number of solutions in each generation is $N$, the crossover probability is $p_{c}$, the mutation probability is $p_{m}$, and the algorithm runs $k$ generations, then the total execution time $T$ is estimated as:

$$
T=t \cdot N \cdot\left(p_{c} \cdot k+p_{m} \cdot k+1\right) .
$$

This is the sum of the calculation time of the initial population and each generation. Since there is no unified theory to determine the crossover and mutation probability, the crossover probability of 0.8 to 1 and the mutation probability of 0.1 to 0.3 have been tried in advance. These experiments show that the probabilities of crossover and mutation within the given ranges have little effect on the optimization results and mainly affect the convergence speed of the algorithm. Based on the results of these experiments, $p_{c}$ and $p_{m}$ are finally set to 1 and 0.2 in the optimization, respectively. The original NSGA-III paper [33] suggests setting the value of $N$ close to the number of reference points. In this optimization, the population size is set to 300 which is sufficient to depict the Pareto-optimal front and the $k$ is set to 100 to ensure convergence. A workstation with two E5-2687W v3 processors and ten cores per processor is used to run this optimization. The execution time of this optimization is about seven days. This execution time will decrease when computing power is increased.

\section{B. Optimization results}

Based on the reference points generated by Das and Dennis's systematic approach, the results of the NSGA-III optimization are shown in Figs. 3 and 4. In the last generation of the NSGA-III optimization, all 300 solutions

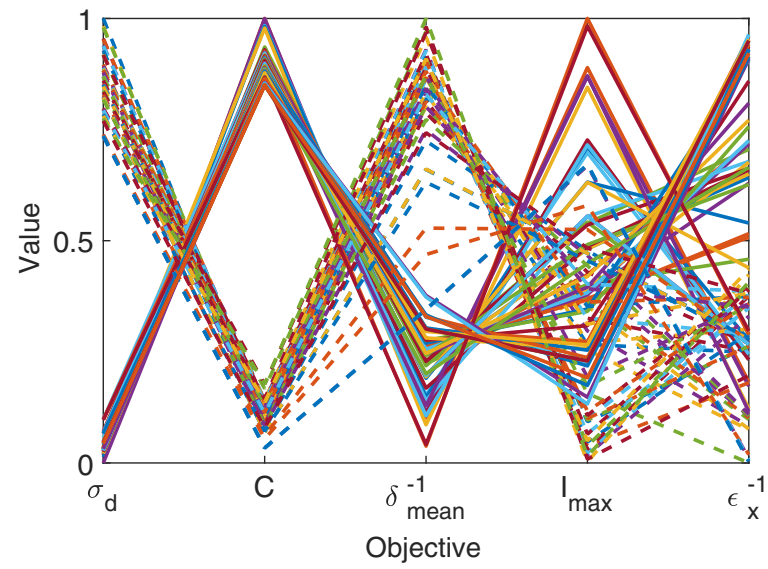

FIG. 3. Parallel coordinate plots of the 50 solutions with the largest energy chirp (dashed lines) and the 50 solutions with the largest current profile factor (solid lines) in the last generation.
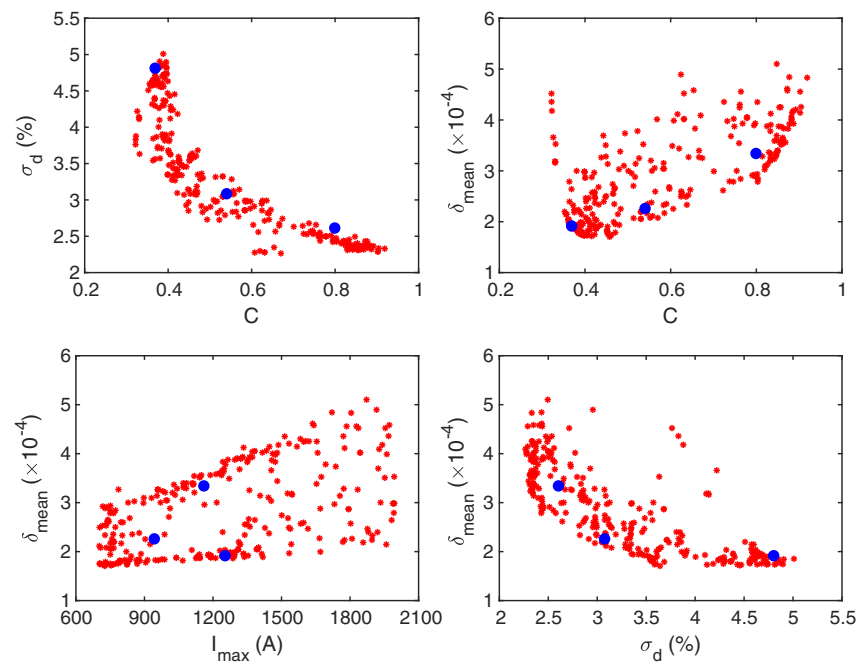

FIG. 4. Projection of the Pareto-optimal front obtained by the NSGA-III on different planes. The three blue dots are three typical solutions whose current profiles and longitudinal phase spaces are presented in Fig. 5.

are on the Pareto-optimal front. Figure 3 shows the 50 solutions with the largest energy chirp and the 50 solutions with the largest current profile factor on the Pareto-optimal front obtained in the last generation, where all the solutions are normalized as follows:

$$
f_{n i j}=\frac{f_{i j}-\min \left(f_{i}\right)}{\max \left(f_{i}\right)-\min \left(f_{i}\right)},
$$

in which $f_{i j}$ is the $i$ th objective value of the $j$ th solution, $\max \left(f_{i}\right)$ and $\min \left(f_{i}\right)$ are the maximum and minimum values of the $i$-th objective in this generation. The Paretooptimal front shows that there is a strong correlation between some goals. In order to better show these relationships, the projection of the Pareto-optimal front on several planes formed by different objectives is presented in Fig. 4. As Fig. 4 (top left) shows, current profile of the electron bunch has a large impact on the maximum available energy chirp. In general, a worse profile factor can achieve a larger energy chirp. Therefore, the trade-off between the energy chirp and current profile is very important. For those solutions that have the same peak current but a larger profile factor, their average slice current is usually higher, which results in a larger average slice energy spread. Thus, as shown in Fig. 4 (top right) and Fig. 4 (bottom left), an increase in the value of current profile factor or peak current will result in an increase in the average slice energy spread. As presented in Fig. 4 (bottom right), since the large energy chirp corresponds to the poor profile factor, it also corresponds to a small average slice energy spread. In addition to these relationships, there is no clear correlation between other objectives. The beam yaw of most solutions has been well optimized in the last generation. 

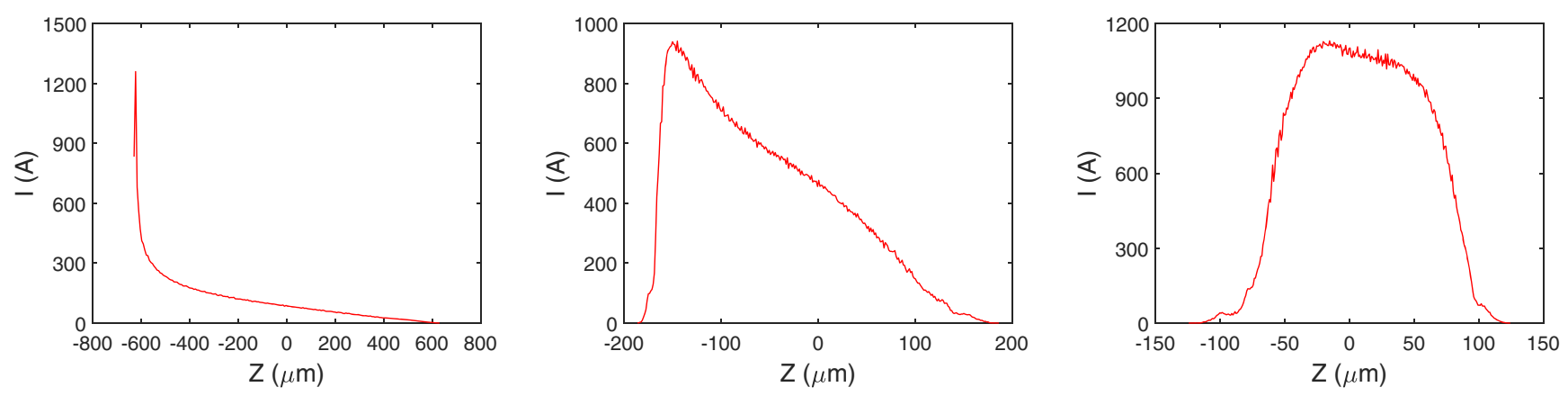

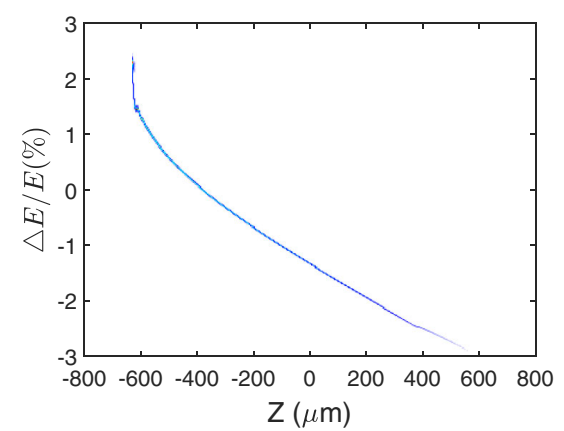

(a) case 1

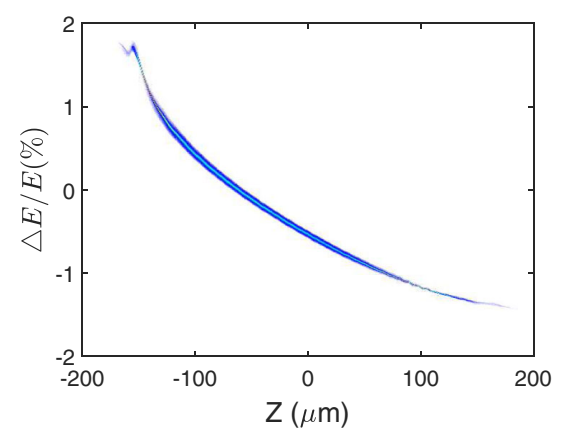

(b) case 2

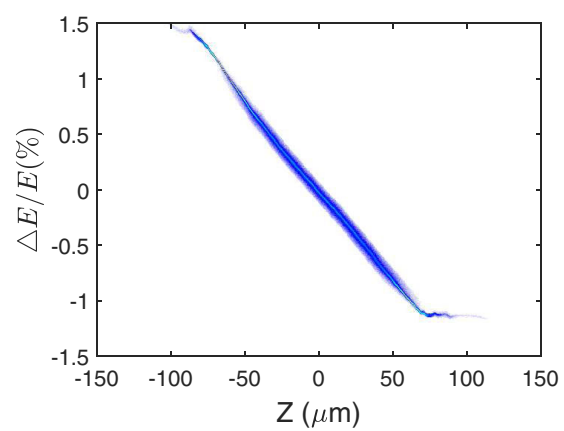

(c) case 3

FIG. 5. Current profile (upper) and longitudinal phase space (lower) of the three typical electron bunches selected from the Paretooptimal front.

From the prospective of the current shape, solutions can be divided into three categories. There are three typical cases from the three kinds of current profile are given in Fig. 4 with blue dots. Fig. 5 shows the current profile and longitudinal phase space of the three cases based on the PELEGANT simulation with one million macroparticles. The objective function values based on one million macroparticles are only slightly different from the values based on one hundred thousand macroparticles. The first kind of electron beams are those with profile factor lower than 0.5 , which have a high-current leading peak and a long tail like the case 1 in Fig. 5(a). The sharp single-horn of this kind of electron beam is due to the nonlinear bunch compression. The maximum energy chirp available in this type of electron beams is 5\%. Although the energy chirp is large, the low-current tail of such kind of electron beams will not contribute to the broad-bandwidth FEL lasing. Therefore, this kind of electron beam is not suitable for the largebandwidth operation mode. The second kind is those electron beams with profile factor between 0.5 and 0.7 . This kind of electron bunch has a quasi-triangular current profile like the case 2 in Fig. 5(b) with a profile factor of 0.54 . Similar to the first kind of electron beams, the lowcurrent tail does not contribute to the FEL lasing which causes the final FEL bandwidth to be much smaller than the theoretical value, i.e., the twice of the energy chirp. In addition, this kind of current shape also leads to a poor uniformity of the power profile. Thus, this kind of electron bunch is not the best choice for producing broad-bandwidth XFEL pulses either.
Those electron beams with a profile factor more than 0.7 are treated as the third kind. The current shape of this kind of electron bunches is more or less flat-top or Gaussian shape, which is suitable for generating largebandwidth FEL pulses to most users. In this kind, electron beams with peak currents between 700 and $2000 \mathrm{~A}$ and similar energy chirp values can be obtained. For those electron beams with peak currents between 700 and $800 \mathrm{~A}$, the beam tilt caused by the CSR is negligible. With the increase of the peak current, the influence of CSR on beam yaw will also increase. Benefit from the beam yaw correction, electron beams with larger peak current can also be used to generate large-bandwidth XFEL pulses. After taking into account the impact of multiple objectives, the case 3 [see Fig. 5(c)] with a peak current of $1150 \mathrm{~A}$ is selected for the large-bandwidth mode of the SXFEL user facility. The energy chirp, normalized horizontal emittance, and profile factor of the chosen electron bunch are $2.61 \%, 1.45 \mathrm{~mm} \cdot \mathrm{mrad}$, and 0.80 . Start-to-end simulations with one million macroparticles have been performed based on the rf parameters of this case. If the quadrupoles in the chicane are not used, there is a clear beam yaw in the horizontal phase space as shown in Fig. 6 (top). As Fig. 6 (bottom) shows, the first order beam yaw in the horizontal phase space has been well corrected. To verify the large-bandwidth generation, the GENESIS simulation runs 20 times with different seeds and the results are shown in Fig. 7. The pulse energy and XFEL bandwidth are $525 \pm 15 \mu \mathrm{J}$ and $4.79 \pm 0.07 \%$ (including a 2\% cut). As shown in Fig. 7 (left), there is 

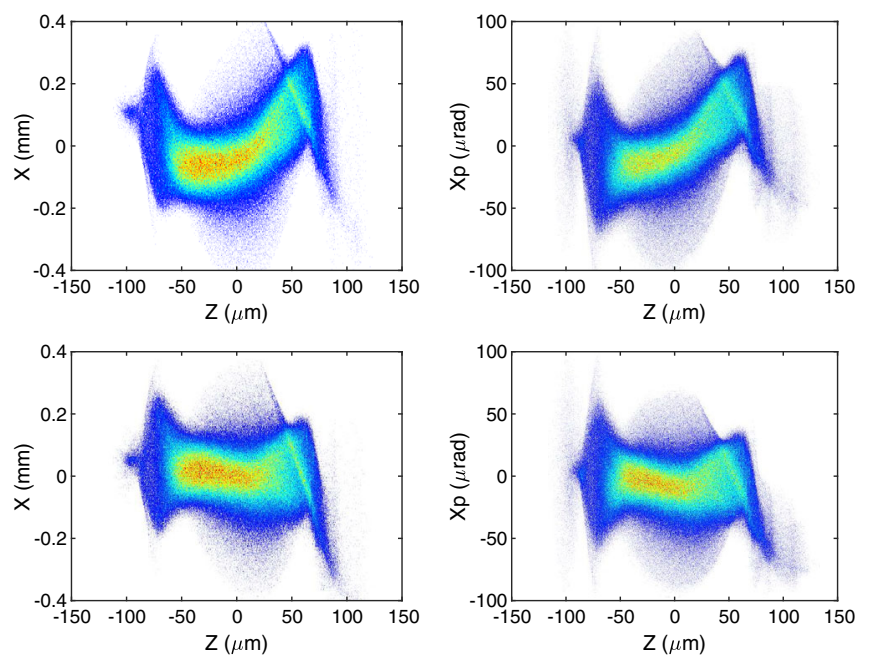

FIG. 6. Uncorrected (top) and corrected (bottom) transverse phase space of the case 3 at the entrance of the undulator.
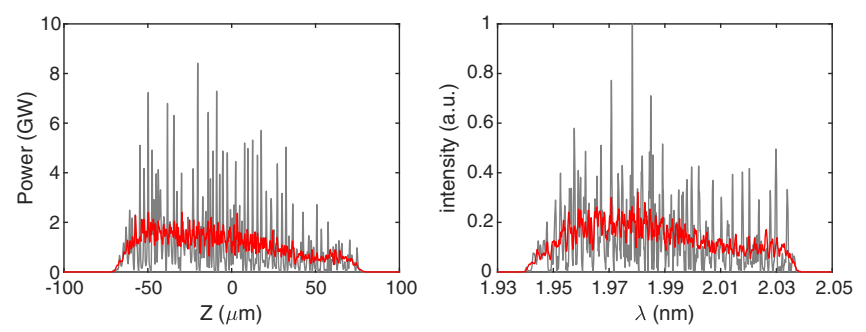

FIG. 7. Simulated FEL power profile (left) and spectrum (right) of the selected electron bunch. The gray lines refer to a single shot and the red lines represent average values of 20 runs with different seeds.

a low radiation power part near $50 \mu \mathrm{m}$ of the XFEL pulse, which is caused by the high-order beam yaw.

To analyze the effects of the voltages and timing jitters in the rf structure, 1000 ELEGANT runs are performed with randomized voltages and phases of the first accelerating section and linearizer. Phase jitters of the S-band and $\mathrm{X}$-band are 0.1 and $0.4 \mathrm{deg}$. Voltage jitters in both sections are $0.04 \%$. The impacts of these jitters on the optimization targets are calculated. The rms jitters of the peak current, profile factor, and energy chirp are $14.18 \%, 2.56 \%$, and $0.90 \%$. The jitter analyses indicate that the FEL bandwidth is stable in the overcompression mode and the peak power may have some fluctuations due to the peak current jitter which is in an acceptable range.

\section{EFFICIENT OPTIMIZATION BASED ON PREFERRED REFERENCE POINTS}

In Sec. III, the method proposed by Das and Dennis is used to generate reference points for the NSGA-III. These reference points are equal for all objectives and are well distributed in the five-dimensional objective space to find the entire Pareto-optimal front and analyze the correlation between the objectives. However, finding the whole Paretooptimal front is usually inefficient and unnecessary, especially for the online optimization of a real machine. For example, to find the entire Pareto-optimal front, solutions that have small energy spread but poor energy chirp and current profile may be retained in the population until the end. Such solutions obviously have no meaning for the large-bandwidth mode and result in longer execution time of the algorithm.

In this study, optimizing the energy chirp and current profile is the most important task while other objectives are also expected to be optimized simultaneously. A feasible way to increase the effect of the values of the energy chirp and profile factor in the optimization is to use only the reference points on the plane formed by the energy chirp and profile factor to guide the optimization. In this case, those solutions with poor energy chirp and profile factor are difficult to preserve in the optimization. Thus, as shown in Fig. 8 (left), only 66 reference points on the plane consisting of energy chirp and profile factor are selected from the reference points generated by the Das and Dennis's method with 10 divisions as a set of preferred reference points. As mentioned before, $-\sigma_{d},-C,-\delta_{\text {mean }}^{-1}$, $-I_{\max }$, and $-\epsilon_{x}^{-1}$ are treated as actual objective functions and they are normalized to a range of 0 to 1 during the optimization. This indicates that the reference point with a smaller value corresponds to the better solution in the optimization. For the other three objectives, the values of these reference points are 0, i.e., the optimal value. To compare the performance of the NSGA-III with preferred reference points [NSGA-III $(\mathrm{P})$ ] with other algorithms, the NSGA-II and the single-objective genetic algorithm (SOGA) are also used to optimize this problem. In the SOGA, the objective is defined as:

$$
F_{S}=c_{1} \cdot \sigma_{d}+c_{2} \cdot I_{\text {max }}+c_{3} \cdot \delta_{\text {mean }}^{-1}+c_{4} \cdot C+c_{5} \cdot \varepsilon_{x}^{-1},
$$

where $c$ is the weight of each subgoal and all these subgoals are normalized according to Eq. (6). The maximum and minimum values of each subgoal in Eq. (6) are approximated by the maximum and minimum values of each objective function in the last generation of NSGA-III. The weight of all sub-goals is set to 1 and the objective
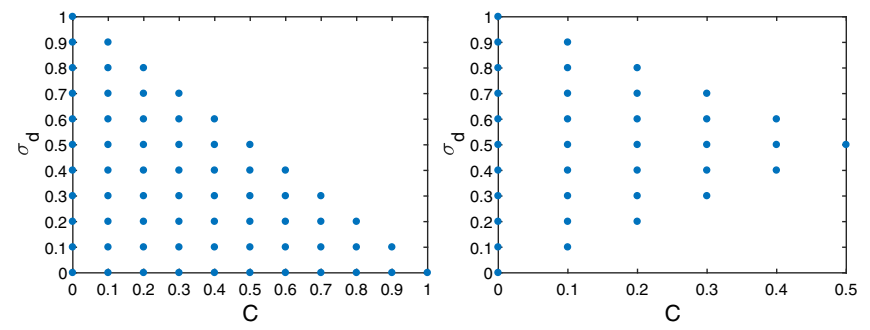

FIG. 8. Two sets of reference points based on different preferences. 
value is maximized in the optimization. For a fair comparison, all algorithms use the same initial population and operation parameters like the mutation and crossover probability. The execution time of NSGA-II and SOGA can still be calculated using Eq. (5). Since the population size and operation parameters of these algorithms are the same, the execution time required can be compared by comparing the generations needed for the convergence of these algorithms.

First, the population size of 300 is considered and the initial population generated in the Sec. III is also used here. The optimization results show that NSGA-II and NSGA-III without preference converge at around the 70th generation and NSGA-III (P) converges at around the 30th generation. The projection of the Pareto-optimal front obtained by the NSGA-III without preference, NSGA-III (P), and NSGA-II on the plane formed by the energy chirp and profile factor in different generations are shown in Fig. 9 and Fig. 10. As shown in Fig. 9, the energy chirp obtained by the NSGA-III (P) is similar to that obtained by the NSGA-III without preference, while the NSGA-III (P) converges faster in regions with good current profile factor. Figure 10 shows that the energy chirp obtained by the NSGA-II in the 100th generation, when the algorithm has converged, is still not as good as that obtained by the NSGA-III (P) in the 30th generation, especially in areas with good current profile factor. As shown in Fig. 10 (bottom right), NSGA-II can obtain some solutions with profile factor very close to 1 and small energy chirp. However, when the profile factor of the solution is in the range of more than 0.7 , the value of the profile factor is not the larger the better for the broadband FEL lasing. In this case, the value of energy chirp is much more important. In addition, the projection of the Pareto-optimal front obtained by the NSGA-III (P) and NSGA-II on other planes are
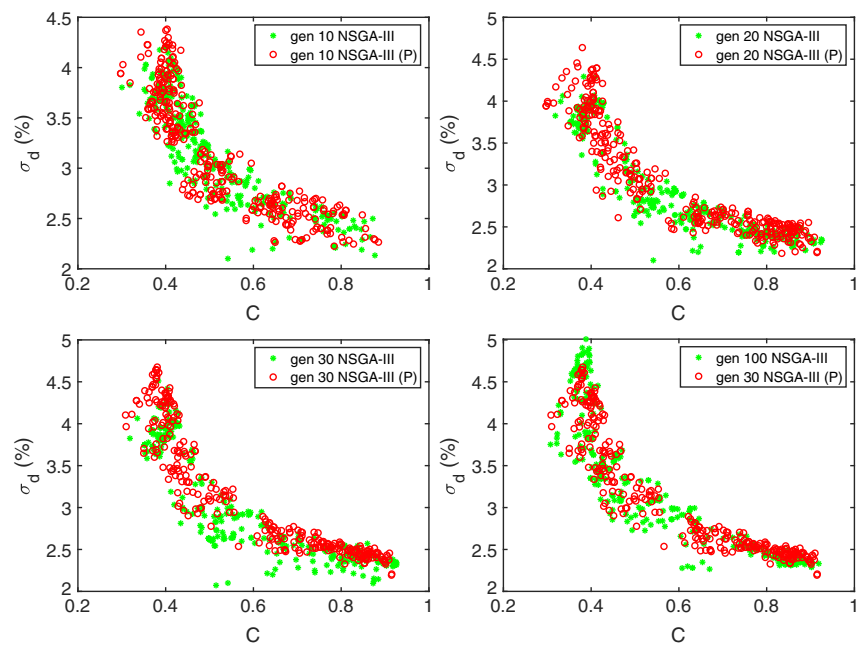

FIG. 9. Projection of the Pareto-optimal front obtained by the NSGA-III and NSGA-III (P) with a population size of 300 in different generations.
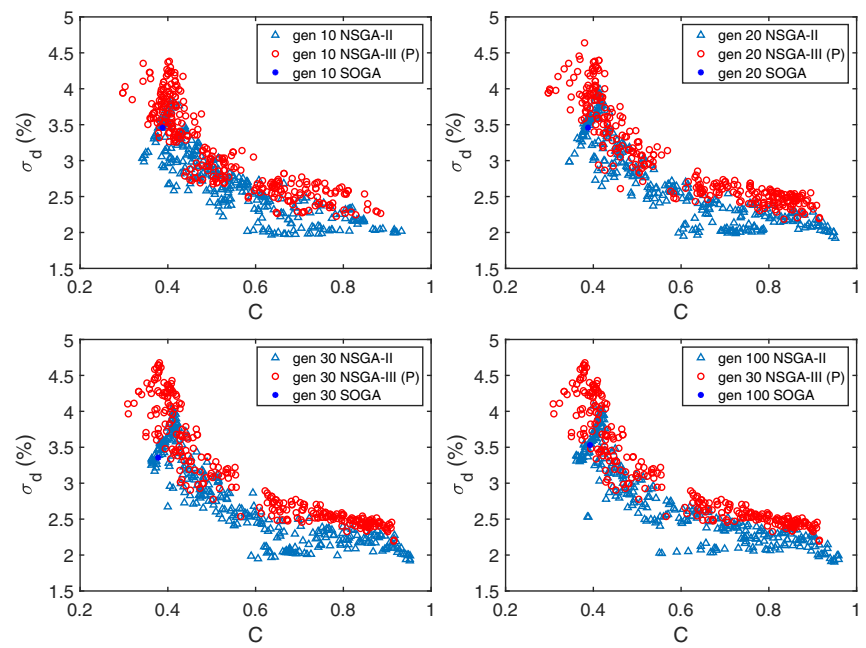

FIG. 10. Projection of the Pareto-optimal front obtained by the NSGA-II and NSGA-III (P) with a population size of 300 in different generations. And the best solutions achieved by the SOGA in different generations.

similar to that obtained by NSGA-III without preference. Therefore, using only the reference points on the plane formed by the energy chirp and profile factor makes the NSGA-III converge faster on the energy chirp and profile factor while the other objective values are not affected. In order to quantitatively compare the results of these algorithms, the average energy chirp of the solutions with a profile factor larger than 0.7 in the last generation of each algorithm is calculated as an evaluation value. In the 30th generation of NSGA-III $(\mathrm{P})$ and the 100th generation of NSGA-III and NSGA-II, the average energy chirp of the solutions with profile factor larger than 0.7 is $2.48 \%$, $2.42 \%$, and $2.21 \%$, respectively. The SOGA converges at around the 40th generation and its optimal value for each generation is on the Pareto-optimal front obtained by the NSGA-III. Moreover, the values of the energy chirp and profile factor of the optimal solution obtained by the SOGA are basically unchanged from the 10th generation. However, the position of the optimal value of the SOGA on the Pareto-optimal front is determined by the weights of the subgoals in the algorithm, which are difficult to determine in advance.

To explore a more efficient optimization, a smaller population size is considered. Here 100 solutions are randomly generated as the initial population and the other parameters in the algorithm are not changed. The results show that NSGA-III (P) and NSGA-II still converge at about the 30th and 70th generation, respectively. In addition, NSGA-II converges to the entire Pareto-optimal front more slowly in the case of small population size. As shown in Fig. 11, NSGA-III (P) converges at around the 30th generation, at this time NSGA-II does not even find a solution with a large current profile factor. The average energy chirp of the solutions having profile factors larger 

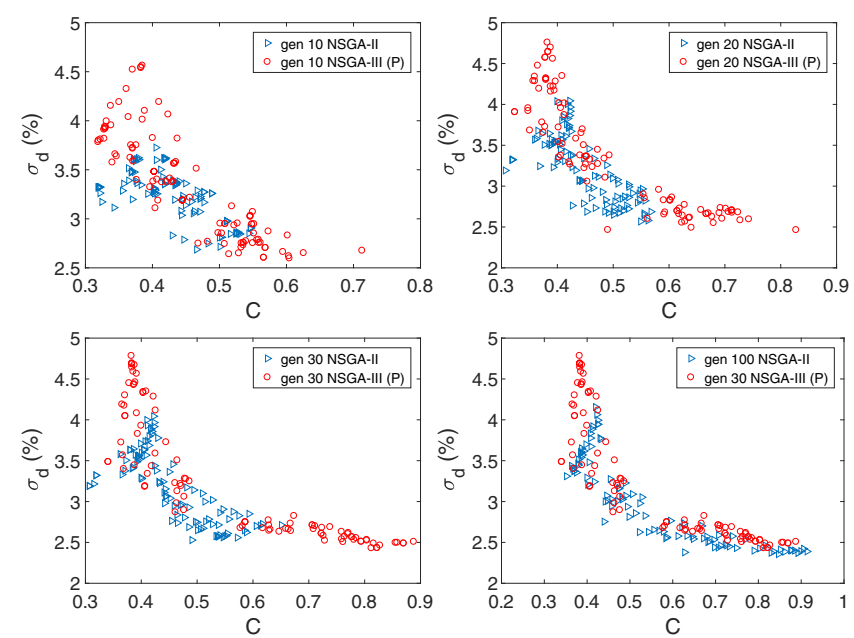

FIG. 11. Projection of the Pareto-optimal front obtained by the NSGA-II and NSGA-III (P) with a population size of 100 in different generations.

than 0.7 in the 30th generation of NSGA-III $(\mathrm{P})$ and the 100th generation of NSGA-II is $2.56 \%$ and $2.43 \%$, respectively. However, when the population size is small, the required part of the Pareto-optimal front, such as a range with a large current profile factor and a specific peak current, there may be only a few solutions for the users to choose from. By further setting constraints on the objectives, more solutions can be concentrated on the preferred area. However, since only a few solutions in previous generations have good profile factors, rejecting solutions with poor profile factors may result in loss of population diversity. In this case, the solution obtained by NSGA-III can be concentrated on the preferred region of the Paretooptimal front by further changing the reference points. In order to achieve this, as presented in Fig. 8 (right), only the upper part of the reference point in Fig. 8 (left) is retained. This is to eliminate the reference points that correspond to solutions with poor profile factors. Therefore, solutions with poor profile factor will not be preserved during the optimization process. To compare with the NSGA-III with the second kind of reference points [NSGA-III (P2)], those solutions with values of profile factor less than 0.7 in the NSGA-II are artificially given the worst objective values. In the optimization, the NSGA-III (P2) and NSGA-II still utilize the same initial population and operation parameters. The corresponding optimization results are presented in Figs. 12 and 13.

The results show that NSGA-III (P2) converges at about the 30th generation while NSGA-II converges at about the 50th generation. Figure 12 shows the projection of the Pareto-optimal front obtained by the NSGA-III (P2) and NSGA-II on the plane formed by the energy chirp and current profile in different generations. The projection of the Pareto-optimal front obtained by the NSGA-III (P2) and NSGA-II on some other planes is presented in Fig. 13. As
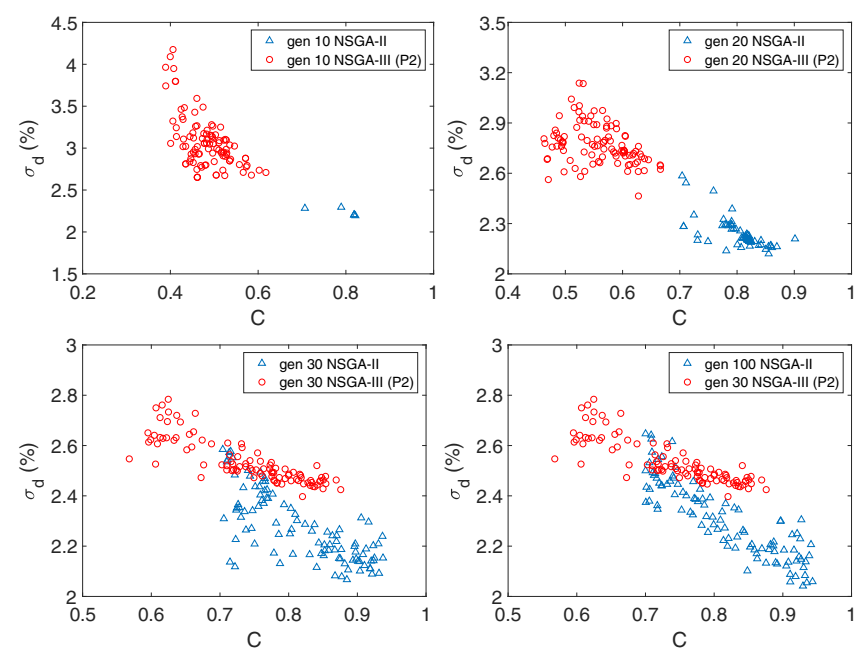

FIG. 12. Projection of the Pareto-optimal front obtained by the NSGA-II (with restrictions on profile factor) and NSGA-III (P2) with a population size of 100 in different generations.

shown in Fig. 12 (top left), only a few solutions on the Pareto-optimal front obtained by NSGA-II in the 10th generation, which means that solutions with a profile factor less than 0.7 account for a high proportion of the population and they are all rejected. The solutions obtained by NSGA-II in the 100th generation are not only inferior to the solutions obtained by NSGA-III (P2) in the 30th generation, but also worse than that obtained by itself without the current profile factor limitation. The average energy chirp of the solutions with a profile factor larger than 0.7 in the 30th generation of NSGA-III (P2) and the 100th generation of NSGA-II is $2.54 \%$ and $2.31 \%$, respectively. The main reason for the deterioration of the NSGA-II optimization results is that a large number of solutions are rejected due to the limitation of the current profile factor, which
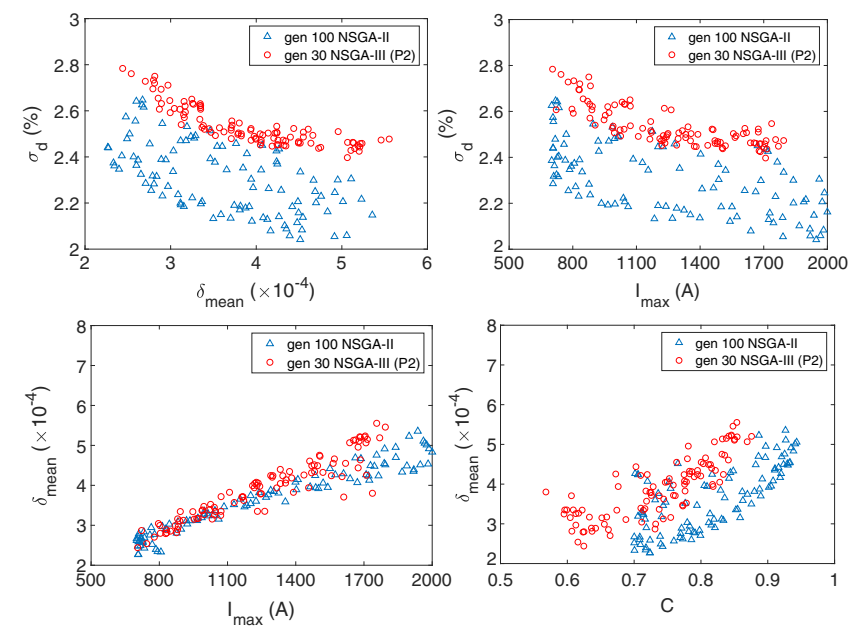

FIG. 13. Projection of the Pareto-optimal front obtained by the NSGA-II (with restrictions on profile factor) and NSGA-III (P2) with a population size of 100 on different planes. 
makes the algorithm falls into local optima. Therefore, when infeasible solutions constitute a large proportion of the entire search space, setting reference points with preferences is more efficient than artificially changing the objective values of those undesired solutions. It should be noted that setting reference points with preference usually only determines the optimization tendency rather than a clear range, so there are some solutions obtained by NSGA-III (P2) with profile factor less than 0.7. In addition, limiting the peak current to a certain range and rejecting electron beams that lack of overcompression still depends on artificially changing the corresponding objective values in this optimization. How to concentrate the entire population in a specific area by setting only special reference points requires further exploration.

From the perspective of online experiments, the execution time of this optimization strategy can also be estimated according to the Eq. (5). And the simulation time of PELEGANT in the equation is replaced by the operation time of the real machine. Since the measurement of the projection emittance in the experiment is very timeconsuming, the projection emittance is not taken into consideration in the online optimization at first. The beam yaw correction is performed after other objectives are well optimized. The other four objective values can be obtained very quickly by reconstructing the longitudinal phase space of the electron bunch with a transverse deflecting structure. The most time-consuming part of this optimization on the SXFEL user facility is to adjust the voltage of the accelerator sections, which takes about 15 seconds each time. If the NSGA-III (P2) is used (the population size is set to 100 and runs for 30 generations) and various accidents such as the rf breakdown are not considered, it takes about 15.4 hours to perform this optimization.

\section{CONCLUSIONS AND OUTLOOK}

In this paper, an evolutionary many-objective optimization algorithm has been applied to optimize the overcompression mode in the linac for producing largebandwidth XFEL pulses. Benefiting from its ability that can optimize more than three goals simultaneously, objectives including the energy chirp, slice energy spread, peak current, current profile, and horizontal projected emittance are all considered in the optimization. In the case of SXFEL user facility, simulations show that the current profile of the electron bunch has a large impact on the maximum available energy chirp. A maximum energy chirp of 5\% can be obtained when the electron beam is of poor current uniformity. Considering the broadband FEL lasing and the dependencies between these objectives, our results indicate that the electron beam with $2.61 \%$ energy chirp generates a $2 \mathrm{~nm}$ FEL pulse with full bandwidth of $4.79 \%$. Furthermore, the preference-based NSGA-III is found to be very efficient at finding preferred parts of the Paretooptimal front. Therefore, this algorithm can be utilized not only for simulation-based design but also for online experiments.

The accuracy of the optimization result largely depends on the definition and calculation method of the optimization objectives. In this paper, the 1D CSR model of the ELEGANT may not be accurate enough for the simulation of compressing electron bunches to large peak currents with single-stage compression. This requires further investigation with more complicated 3D CSR simulations. Moreover, it is also possible to optimize the problem based on the GENESIS simulation results when the computing power is sufficient.

This optimization strategy can be easily extended to other problems, especially for various XFEL operation modes that need to find the optimal working point of the linac, such as the twin bunch operation mode [51] and nonlinear compression mode [52,53]. In addition, this optimization strategy can also be used for the electron bunch shaping using the entire accelerator, such as generating a triangular electron beam to improve the transformer ratio in the beam-driven collinear wakefield accelerators $[54,55]$.

\section{ACKNOWLEDGMENTS}

The author would like to thank Z. Wang, M. Zhang, B. Liu, D. Wang and Z. Zhao for helpful discussions on beam dynamics and SXFEL projects; J. G. Power for useful discussions on beam-driven collinear wakefield accelerators. This work was partially supported by the National Natural Science Foundation of China (11775293), the National Key Research and Development Program of China (2016YFA0401900), the Young Elite Scientist Sponsorship Program by CAST (2015QNRC001) and Ten Thousand Talent Program.

[1] C. Pellegrini, A. Marinelli, and S. Reiche, The physics of X-ray free-electron lasers, Rev. Mod. Phys. 88 (2016).

[2] P. Emma, R. Akre, J. Arthur, R. Bionta, C. Bostedt, J. Bozek, A. Brachmann, P. Bucksbaum, R. Coffee, F.-J. Decker et al., First lasing and operation of an ångstromwavelength free-electron laser, Nat. Photonics 4, 641 (2010).

[3] W. a. Ackermann, G. Asova, V. Ayvazyan, A. Azima, N. Baboi, J. Bähr, V. Balandin, B. Beutner, A. Brandt, A. Bolzmann et al., Operation of a free-electron laser from the extreme ultraviolet to the water window, Nat. Photonics 1, 336 (2007).

[4] D. Pile, First light from SACLA, Nat. Photonics 5, 456 (2011).

[5] E. Allaria, D. Castronovo, P. Cinquegrana, P. Craievich, M. D. Forno, M. B. Danailov, G. D'Auria, A. Demidovich, G. D. Ninno, and S. D. Mitri, Two-stage seeded soft-X-ray free-electron laser, Nat. Photonics 7, 913 (2013).

[6] H. S. Kang, C. K. Min, H. Heo, C. Kim, H. Yang, G. Kim, I. Nam, S. Y. Baek, H. J. Choi, and G. Mun, Hard X-ray 
free-electron laser with femtosecond-scale timing jitter, Nat. Photonics 11, 708 (2017).

[7] A. Kondratenko and E. Saldin, Generating of coherent radiation by a relativistic electron beam in an ondulator, Part. Accel. 10, 207 (1980).

[8] R. Bonifacio, C. Pellegrini, and L. Narducci, Collective instabilities and high-gain regime in a free electron laser, Opt. Commun. 50, 373 (1984).

[9] C. Feng and H.-X. Deng, Review of fully coherent freeelectron lasers, Nucl. Sci. Tech. 29, 160 (2018).

[10] B. D. Patterson, R. Abela, H. H. Braun, U. Flechsig, R. Ganter, Y. Kim, E. Kirk, A. Oppelt, M. Pedrozzi, and S. Reiche, Coherent science at the SwissFEL X-ray laser, New J. Phys. 12, 035012 (2010).

[11] S. Baradaran, U. Bergmann, H. Durr, K. Gaffney, J. Goldstein, M. Guehr, J. Hastings, P. Heimann, R. Lee, M. Seibert et al., LCLS-II new instruments workshops report, SLAC National Accelerator Lab., Technical Report No. SLAC-R-993, 2012.

[12] W. A. Hendrickson and C. M. Ogata, [28] Phase determination from multiwavelength anomalous diffraction measurements, Methods Enzymol. 276, 494 (1997).

[13] S.-K. Son, H. N. Chapman, and R. Santra, Multiwavelength Anomalous Diffraction at High X-Ray Intensity, Phys. Rev. Lett. 107, 218102 (2011).

[14] C. Dejoie, L. B. Mccusker, C. Baerlocher, R. Abela, B. D. Patterson, M. Kunz, and N. Tamura, Using a nonmonochromatic microbeam for serial snapshot crystallography, J. Appl. Crystallogr. 46, 791 (2013).

[15] C. Dejoie, S. Smeets, C. Baerlocher, N. Tamura, P. Pattison, R. Abela, and L. B. McCusker, Serial snapshot crystallography for materials science with SwissFEL, IUCrJ 2, 361 (2015).

[16] E. Prat, M. Calvi, and S. Reiche, Generation of ultra-largebandwidth X-ray free-electron-laser pulses with a transverse-gradient undulator, J. Synchrotron Radiat. 23, 874 (2016).

[17] M. Song, J. Yan, K. Li, C. Feng, and H. Deng, Bandwidth broadening of X-ray free electron laser pulses with the natural gradient of planar undulator, Nucl. Instrum. Methods Phys. Res. 884, 11 (2018).

[18] S. Serkez, V. Kocharyan, E. Saldin, I. Zagorodnov, G. Geloni, and O. Yefanov, Proceedings of FEL New York, NY, USA (JACoW, New York, NY, USA, 2013), p. 345, Vol. 2.

[19] P. Emma, Chirping the LCLS Electron Beam, SLAC National Accelerator Lab. Technical Report No. LCLSTN-00-6, 2000.

[20] A. S. Hernandez, E. Prat, S. Bettoni, B. Beutner, and S. Reiche, Generation of large-bandwidth X-ray free-electronlaser pulses, Phys. Rev. Accel. Beams 19, 090702 (2016).

[21] J. L. Turner, F. Decker, Y. Ding, Z. Huang, R. Iverson, J. Krzywinski, H. Loos, A. Marinelli, T. Maxwell, H. Nuhn et al., in Proceedings of the 36th International Free Electron Laser Conference Basel, Switzerland (JACoW, Basel, Switzerland, 2014), p. 337.

[22] M. Guetg, F.-J. Decker, Y. Ding, P. Emma, Z. Huang, and T. Maxwell, in 7th International Particle Accelerator Conference (IPAC'16), Busan, Korea, 2016 (JACOW, Geneva, Switzerland, 2016), pp. 813-816.
[23] I. Zagorodnov, G. Feng, and T. Limberg, Corrugated structure insertion for extending the SASE bandwidth up to $3 \%$ at the European XFEL, Nucl. Instrum. Methods Phys. Res., Sect. A 837, 69 (2016).

[24] A. Konak, D. W. Coit, and A. E. Smith, Multi-objective optimization using genetic algorithms: A tutorial, Reliability Engineering \& System Safety 91, 992 (2006).

[25] K. Deb, A. Pratap, S. Agarwal, and T. Meyarivan, A fast and elitist multiobjective genetic algorithm: NSGA-II, IEEE Trans. Evol. Comput. 6, 182 (2002).

[26] I. V. Bazarov and C. K. Sinclair, Multivariate optimization of a high brightness dc gun photoinjector, Phys. Rev. ST Accel. Beams 8, 034202 (2005).

[27] R. Bartolini, M. Apollonio, and I. Martin, Multiobjective genetic algorithm optimization of the beam dynamics in linac drivers for free electron lasers, Phys. Rev. ST Accel. Beams 15, 030701 (2012).

[28] W. Gao, L. Wang, and W. Li, Simultaneous optimization of beam emittance and dynamic aperture for electron storage ring using genetic algorithm, Phys. Rev. ST Accel. Beams 14, 094001 (2011).

[29] J. Wu, N. Hu, H. Setiawan, X. Huang, T. O. Raubenheimer, Y. Jiao, G. Yu, A. Mandlekar, S. Spampinati, K. Fang et al., Multi-dimensional optimization of a terawatt seeded tapered Free Electron Laser with a Multi-Objective Genetic Algorithm, Nucl. Instrum. Methods Phys. Res., Sect. A 846, 56 (2017).

[30] A. Hofler, B. Terzić, M. Kramer, A. Zvezdin, V. Morozov, Y. Roblin, F. Lin, and C. Jarvis, Innovative applications of genetic algorithms to problems in accelerator physics, Phys. Rev. ST Accel. Beams 16, 010101 (2013).

[31] H. Ishibuchi, N. Akedo, and Y. Nojima, Behavior of multiobjective evolutionary algorithms on many-objective knapsack problems, IEEE Trans. Evol. Comput. 19, 264 (2015).

[32] B. Li, J. Li, K. Tang, and X. Yao, Many-objective evolutionary algorithms: A survey, ACM Computing Surveys (CSUR) 48, 13 (2015).

[33] K. Deb and H. Jain, An evolutionary many-objective optimization algorithm using reference-point-based nondominated sorting approach, part I: Solving problems with box constraints, IEEE Trans. Evol. Comput. 18, 577 (2014).

[34] H. Jain and K. Deb, An evolutionary many-objective optimization algorithm using reference-point based nondominated sorting approach, part II: handling constraints and extending to an adaptive approach, IEEE Trans. Evol. Comput. 18, 602 (2014).

[35] M. W. Mkaouer, M. Kessentini, S. Bechikh, K. Deb, and M. Ó. Cinnéide, in Proceedings of the 2014 Annual Conference on Genetic and Evolutionary Computation (ACM, Vancouver, BC, Canada, 2014), pp. 1263-1270.

[36] M. Tavana, Z. Li, M. Mobin, M. Komaki, and E. Teymourian, Multi-objective control chart design optimization using NSGA-III and MOPSO enhanced with DEA and TOPSIS, Expert Systems with Applications 50, 17 (2016).

[37] X. Yuan, H. Tian, Y. Yuan, Y. Huang, and R. M. Ikram, An extended NSGA-III for solution multi-objective hydrothermal-wind scheduling considering wind power cost, Energy Conversion and Management 96, 568 (2015). 
[38] K. Flöttmann et al., Astra: A space charge tracking algorithm[J]. Manual, Version, 2011, 3, 2014

[39] M. Borland, ANL Advanced Photon Source, Argonne National Lab. Report No. LS-287, 2000.

[40] Y. Wang and M. Borland, Pelegant: A parallel accelerator simulation code for electron generation and tracking, AIP Conf. Proc. 877, 241 (2006).

[41] S. Reiche, Pelegant: A parallel accelerator simulation code for electron generation and tracking, Nucl. Instrum. Methods Phys. Res., Sect. A 429, 243 (1999).

[42] D. Kalyanmoy et al., Multi Objective Optimization Using Evolutionary Algorithms (John Wiley and Sons, New York, 2001).

[43] R. B. Agrawal, K. Deb, and R. Agrawal, Simulated binary crossover for continuous search space, Complex Syst. 9, 115 (1995).

[44] S. Kukkonen and K. Deb, in 2006 IEEE International Conference on Evolutionary Computation (IEEE, Vancouver, BC, Canada, 2006), pp. 1179-1186.

[45] I. Das and J. E. Dennis, Normal-boundary intersection: A new method for generating the pareto surface in nonlinear multicriteria optimization problems, SIAM J. Optim. 8, 631 (1998).

[46] Z. Zhao, D. Wang, Q. Gu, L. Yin, M. Gu, Y. Leng, and B. Liu, Status of the SXFEL Facility, Appl. Sci. 7, 607 (2017).

[47] E. L. Saldin, E. A. Schneidmiller, and M. Yurkov, Nucl. Instrum. Methods Phys. Res., Sect. A 398, 373 (1997).

[48] M. W. Guetg, B. Beutner, E. Prat, and S. Reiche, Optimization of free electron laser performance by dispersionbased beam-tilt correction, Phys. Rev. ST Accel. Beams 18, 030701 (2015).
[49] M. W. Guetg, A. A. Lutman, Y. Ding, T. J. Maxwell, F. J. Decker, U. Bergmann, and Z. Huang, Generation of High-Power High-Intensity Short X-Ray Free-ElectronLaser Pulses, Phys. Rev. Lett. 120, 014801 (2018).

[50] C. A. Coello Coello, A survey of constraint handling techniques used with evolutionary algorithms, Laboratorio Nacional de Informática Avanzada, Technical Report No. Lania-RI-99-04, 1999.

[51] A. Marinelli, D. Ratner, A. Lutman, J. Turner, J. Welch, F.-J. Decker, H. Loos, C. Behrens, S. Gilevich, A. Miahnahri et al., High-intensity double-pulse X-ray freeelectron laser, Nat. Commun. 6, 6369 (2015).

[52] S. Huang, Y. Ding, Z. Huang, and J. Qiang, Generation of stable subfemtosecond hard x-ray pulses with optimized nonlinear bunch compression, Phys. Rev. ST Accel. Beams 17, 120703 (2014).

[53] S. Huang, Y. Ding, Y. Feng, E. Hemsing, Z. Huang, J. Krzywinski, A. A. Lutman, A. Marinelli, T. J. Maxwell, and D. Zhu, Generating Single-Spike Hard X-Ray Pulses with Nonlinear Bunch Compression in Free-Electron Lasers, Phys. Rev. Lett. 119, 154801 (2017).

[54] P. Piot, C. Behrens, C. Gerth, M. Dohlus, F. Lemery, D. Mihalcea, P. Stoltz, and M. Vogt, Generation and Characterization of Electron Bunches with Ramped Current Profiles in a Dual-Frequency Superconducting Linear Accelerator, Phys. Rev. Lett. 108, 034801 (2012).

[55] Q. Gao, G. Ha, C. Jing, S. Antipov, J. Power, M. Conde, W. Gai, H. Chen, J. Shi, E. Wisniewski et al., Observation of High Transformer Ratio of Shaped Bunch Generated by an Emittance-Exchange Beam Line, Phys. Rev. Lett. 120, 114801 (2018). 